\title{
Developing an Adaptive Evacuation Simulation Framework based on Agent Performance Dynamics
}

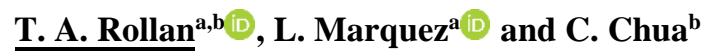 \\ ${ }^{a}$ CSIRO Data61, Clayton, Australia, ${ }^{b}$ Swinburne University of Technology, Hawthorn, Australia
}

Email: trollan@swin.edu.au

\begin{abstract}
The benefits of evacuation drills for disaster preparedness are not yet well-established. Virtual reality and serious games (SG) application to evacuation simulation were proven to be effective in overcoming limitations of traditional emergency training approaches with a significant increase in the trainees' knowledge and self-efficacy. Further, human behavioural modelling is a critical component of evacuation simulation.
\end{abstract}

This study will develop an evacuation simulation framework, incorporating various approaches such as cluster analysis, structural equation modelling, agent-based modelling, cognitive modelling and serious games. Although the case study focuses on earthquake, the components of the evacuation framework is designed to be applicable to other types of disaster requiring evacuation. The study's contributions include 1) a cognitive agent model, incorporating experiences, personality, emotions and behavioural decision-making, 2) an implementation of the behavioural modification approach as the foundation for efficient evacuation, 3) a reproduction of a multi-storey structure to add layers of complexity to the evacuation, and 4) a decision support tool to evaluate existing strategies and propose new strategies that will adapt to agent performance dynamics.

The study site is a university with a student population of approximately 8000 , located at around $8.4 \mathrm{~km}$ from a major earthquake fault line and lacks open spaces for evacuees and emergency responders. Therefore, the site is significantly at high risk for a large number of casualties once the anticipated earthquake of extreme magnitude with a minimum of 7.2 strikes. Four scenarios are being considered including Scenario 1 which represents a regular enrolment day when student numbers are expected to be moderately higher than a regular school day. Scenario 2 represents the peak time slot on a regular school day when the population is at its maximum. Scenarios 3 and 4 are both days with extra-curricular events when the number of visitors is at its maximum.

An online earthquake evacuation survey was designed to conduct population profiling for the occupants of the university. Cluster analysis will divide the survey participant population into evacuee groups in terms of their demographic information, personality traits and their responses to questions on past and future behaviour and decision-making during evacuations. Preliminary implementation in Unity game development software involved simulation runs using an initial version of the agents being aware of the exit locations. For each timestamp, the simulation engine stores information per agent including a generated unique agent number ID, role including faculty and student, current location such as first floor and parking lot, 3D coordinates and the evacuation state. The evacuation simulation component will produce evacuee count vs time and evacuee floor level vs time graphs, and evacuee route and heat maps. The decision support component will produce a set of proposed evacuee schedule and route assignments. The proposal for the evacuee population profile composition will be based on the experiments that will be conducted focusing on how behavioural modification can improve evacuation performance.

The succeeding version of the implementation will apply the results of the survey on the agent model and perform the simulations on the four identified crucial scenarios.

Keywords: Evacuation simulation, agent-based modelling, behavioural modelling, emergency decision support, disaster management 


\section{INTRODUCTION}

The unpredictability of disasters renders preparedness and time efficiency as highly valued factors in order to minimize injuries and casualties. Both objectives can be fulfilled by utilizing existing tools and exploring emerging information technologies for proper planning and implementation of evacuation. Evacuation drills, as the premeditated course of action to improve and assess performance, are still under scrutiny with regards to their impact on actual evacuations (Gwynne et al., 2017). The benefits of evacuation drills are being questioned considering their inherent risk to participants, significant execution costs, inconsistencies in terms of training and performance assessment (e.g. exclusion of physically disabled individuals), adaptability to constantly evolving scenarios and population demographics, and manual collection of potentially subjective data (Gwynne et al., 2020).

According to Feng et al., traditional training approaches such as seminars, posters, videos or drills need to be improved because these methods tend to be less engaging and lack the sensory capabilities to imitate life threatening situations (Feng et al., 2020). The authors' accomplishment on the creation of training tools using immersive virtual reality (IVR) and serious games (SG) were proven to be effective in overcoming these limitations with a significant increase in the trainees' knowledge and self-efficacy. SG was also applied by García-García et al. to formulate a personality-driven behaviour model for emergency evacuation (GarcíaGarcía et al., 2013). A two-dimensional SG for earthquake preparation and handling demonstrated better learning capability than reading materials (Chen, 2015).

This study focuses on the development of an evacuation simulation framework that will act as a guide for developing tools for the evaluation of evacuation strategies in collaboration with decision-making authorities. Its first contribution is a representative human agent, incorporating the possible effects of demographic information and past disaster experiences on personality, emotions and behaviours. Its second contribution is an implementation of the behavioural modification approach as the foundation for efficient evacuation, and for the review and improvement of safety protocols and practices. Compared to architectural and path/schedule planning solutions, this underexplored approach offers new possibilities in practicality and ease of implementation by viewing people as the solution (Haghani, 2020). Its third contribution is a reproduction of a multi-storey structure to add layers of complexity to the evacuation (Choi and Do, 2019; Rendón Rozo et al., 2019; Sun et al., 2019). Finally, its fourth contribution is a decision support tool designed to evaluate existing evacuation plans and propose new evacuation strategies that will adapt to the dynamics of the agents' evacuation performance.

The study site is a university with a student population of approximately 8000 . It is situated around $8.4 \mathrm{~km}$ from a major earthquake fault line and lacks open spaces for evacuees and emergency responders. Therefore, the site is significantly at high risk for a large number of casualties once the anticipated earthquake of extreme magnitude with a minimum of 7.2 (Velasco et al., 2019) strikes.

\section{THE EVACUATION SIMULATION FRAMEWORK}

There are three main components in the evacuation simulation framework illustrated in Figure 1 namely the Cognitive Agent Model using SPSS Statistics and AMOS, the Evacuation Simulation using Unity game development platform and the Decision Support using Power BI.

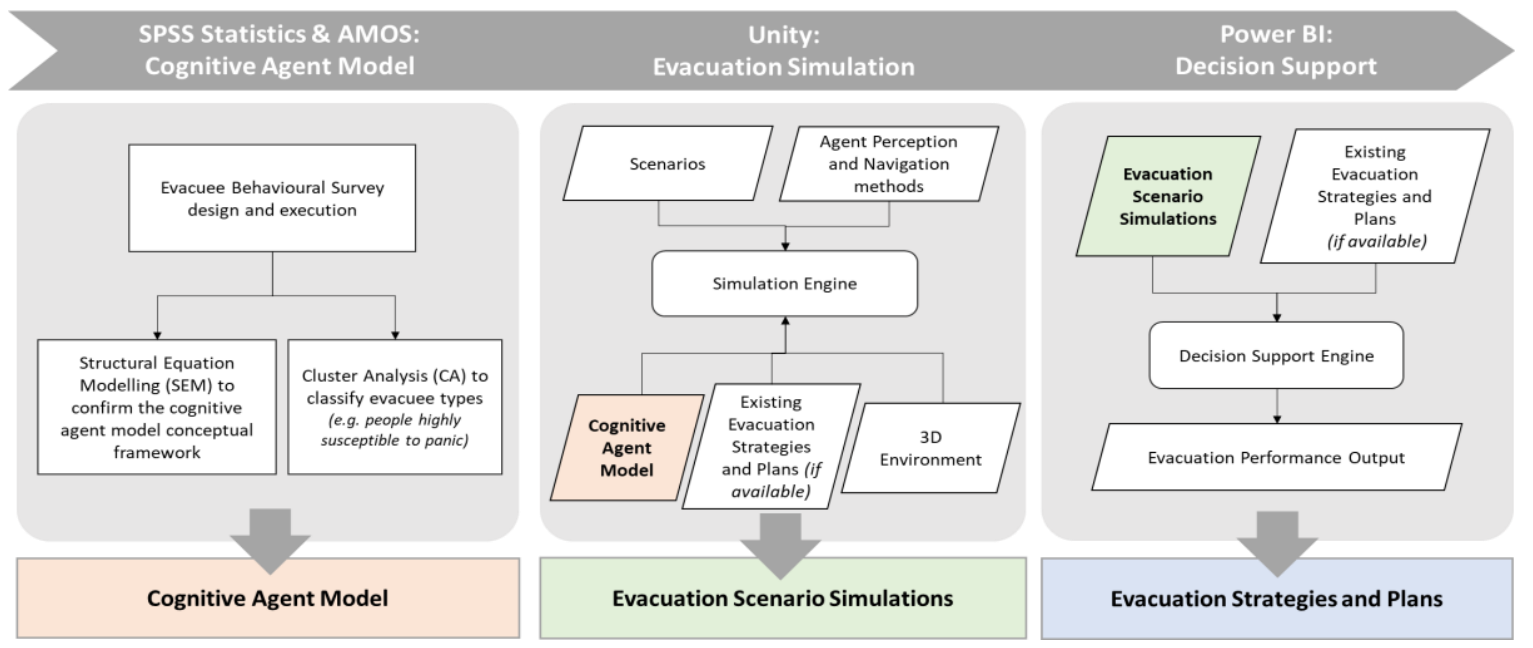

Figure 1. The Evacuation Simulation Framework 
In Section 2.1, the first component involving the formulation of the cognitive agent model includes the design and conduct of an earthquake evacuation behavioural survey, cluster analysis and structural equation modelling. The resulting cognitive agent model will then be used in the second component which is the evacuation simulation. Among the inputs to the simulation engine are the scenarios which are further discussed in Section 2.2, the perception and navigation methods with the main references described in Section 2.3, the existing evacuation strategies and plans including route and assembly maps, and the threedimensional environment with snapshots shown in the preliminary implementation in Section 3 which were based on floorplans and reference site images. The main strategies to improve evacuation performance that will guide the decision support component are discussed in Section 2.4. The inputs to this third component include the evacuation scenario simulations obtained from the second component and the existing evacuation strategies and plans while the evacuation performance outputs will include the evacuation data statistics, tables, graphs, and maps. The decision support evaluation process will be iterative until a suitable primary evacuation strategy is achieved.

The following sections further discuss the details of the components of the evacuation simulation framework.

\subsection{Cognitive Agent Model}

An online earthquake evacuation survey was designed including questions to measure spatial familiarity of the study site, earthquake evacuation protocol and routes, dimensions and survival-oriented personality traits, emotion contagion and herd behaviour tendencies, and behavioural decision-making. The survey was conducted to the occupants of the university from which a total of 579 responses were obtained. The participants are comprised of $68 \%$ female, $27 \%$ male and the remaining belong to the non-binary group or preferred not to disclose their gender. A large fraction of the responses, specifically $73 \%$, come from the students while the rest are from faculty and staff. Only $4 \%$ of the participants have medical or safety and security backgrounds. The majority (84\%) have already experienced an evacuation drill. Only 5\% have not yet experienced either an evacuation drill or an earthquake.

Cluster analysis will divide the survey participant population into evacuee groups (archetypes) in terms of their demographic information, personality traits and their responses to questions on past and future behaviour and decision-making during evacuations. Confirmatory factor analysis (CFA), facilitated by structural equation modelling (SEM) using IBM AMOS software, will be used to determine whether the paths and dependencies illustrated by the conceptual framework in Figure 2 are deemed appropriate.

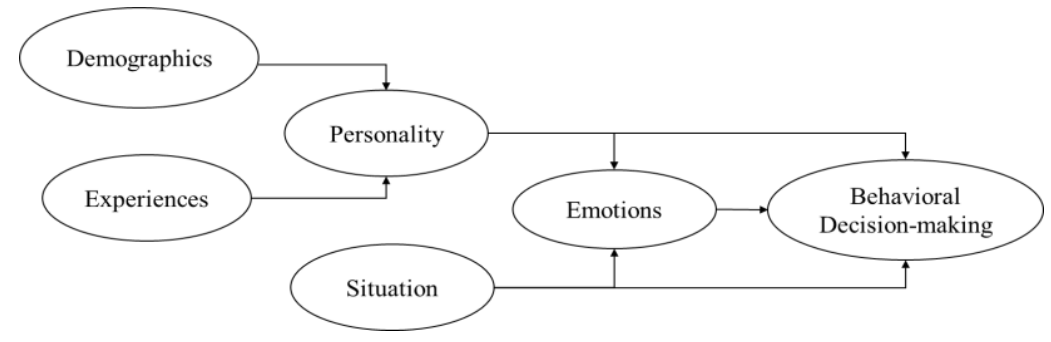

Figure 2. Conceptual Framework for CFA - SEM

This conceptual framework suggests the direct influence of both demographic information and disasterrelated experiences on one's personality. Further, the conceptual framework proposes both partial direct effect of personality and situation to one's emotional state. According to SEM, personality and situation will have partial direct effects on behavioural decision-making with or without the mediation of emotions.

\subsection{Simulation Scenarios}

Table 1 summarizes the characteristics and datasets associated with four scenarios identified by the university's representatives. Scenario 1 represents a regular enrolment day when student numbers are expected to be moderately higher than a regular school day. Accompanying some of the students would be visitors who are not familiar with the spatial configuration of the campus. Similarly, first-year students would have the same level of familiarity with the campus as visitors. Scenario 2 represents the peak time slot of 11 am on either a Tuesday or a Thursday when the school population is at its maximum. Scenarios 3 and 4 are both days with extra-curricular events when the number of visitors is at its maximum. In scenario 4 , the basketball court, located on the $8^{\text {th }}$ floor, is used for sports competitions and contains crowds of visitors unfamiliar with emergency routes and exits. 
Table 1. Scenarios and respective datasets to establish them in the simulation

\begin{tabular}{lll}
\hline & Description & Dataset \\
\hline Scenario 1 & Enrolment day & Expected daily enrolee count, Enrolment-related venues \\
Scenario 2 & Peak of regular school day & Class schedule matrix \\
Scenario 3 & Medical mission day & Expected blood donor and visitor count, Event venues \\
Scenario 4 & Sports event day & Expected visitor count, Event participant count and venues \\
\hline
\end{tabular}

\subsection{Perception and Navigation}

Simulation space in Unity is categorized into four types: 1) fixed-feature space with immovable objects such as walls and territorial boundaries), 2) semifixed-feature space with movable objects including pieces of furniture, 3) movement space for agents, and 4) informal space or the personal space around the body of agents (Littlejohn and Foss, 2005; Low and Lawrence-Zúñiga, 2003). Wąs et al. claimed that normally, individuals or groups of people adhere to the concept of territoriality and the characteristics of a classical social zone (Wąs et al., 2012). However, evacuation situations may be different as pedestrians forego territoriality in exchange for the desire to safely escape from the occupied facility using the fastest path or shortest path.

To embed perception and navigation capabilities in agents, they are given visual sensors to continuously collect data from their environment (Şahin and Alhajj, 2020). Zoumpoulaki et al. extended the implementation by giving agents additional visual and aural perception including the ability to detect or sense alarm sounds, other agents, fire, exit signs and exits even when these are out of sight or hearing (Zoumpoulaki et al., 2010). Rahman made use of a constant distance $\Lambda$ of $0.976 \mathrm{~m}$, referred to as the psychological radius to denote a person's tendency to maintain distance from others when walking (Kiyono et al., 1996; Rahman, 2019). At the perception stage, four kinds of information can be tracked by the agent within its simulated perception field namely 1) visible navigation objects, 2) visible group members, 3) neighbouring agents and 4) detected cues (Chu et al., 2015). Ni et al. applied a movement speed ranging from 1.42 to $2.56 \mathrm{~m} / \mathrm{s}$ based on the general walking speed (Mohler et al., 2007; Ni et al., 2018).

\subsection{Decision Support Tool}

The decision support component produces an evaluation of existing evacuation strategies based on the results of the simulation for a given scenario (e.g., routes taken, exits used, assembly areas selected, bottlenecks created). The primary evacuation strategy will be selected based on evaluation criteria put together from consultations with the university's representatives and relevant literature. Song et al. proposed the following strategies to improve evacuation performance (Song et al., 2019):

- Strategy 1: Promotion of earthquake knowledge and placement of more evacuation signs or guides to enhance the population's awareness of the surroundings

- Strategy 2: Improvement of the familiarity towards all exits, corridors, and encouragement on the use of emergency staircases when necessary to weaken specific habitual preferences and add route options

- Strategy 3: Development of an earthquake protocol containing not only fixed instructions (e.g. follow the crowd, evacuate from exit A, etc.) but also guidelines such as to stay calm, identify the most effective route based on the origin location, avoid blind following of the crowd, search for the nearest emergency exit/staircase, and so on

- Strategy 4: Establishment of evacuation plans to reduce interaction among people through effective and accurate information dissemination

\section{PRELIMINARY IMPLEMENTATION IN UNITY}

Figure 3 shows the nine-storey university building, the arrangement of furniture in an office area (lower left) and classrooms (lower right), including walls and partitions. The designation of agents in each area will be based on the university's schedule matrix. Supporting details needed for the evacuation simulation experiments, such as occasional blockage of egress points that will affect the spatial configuration and agent routing, will be supplied through continuous consultation with the university's representatives. 

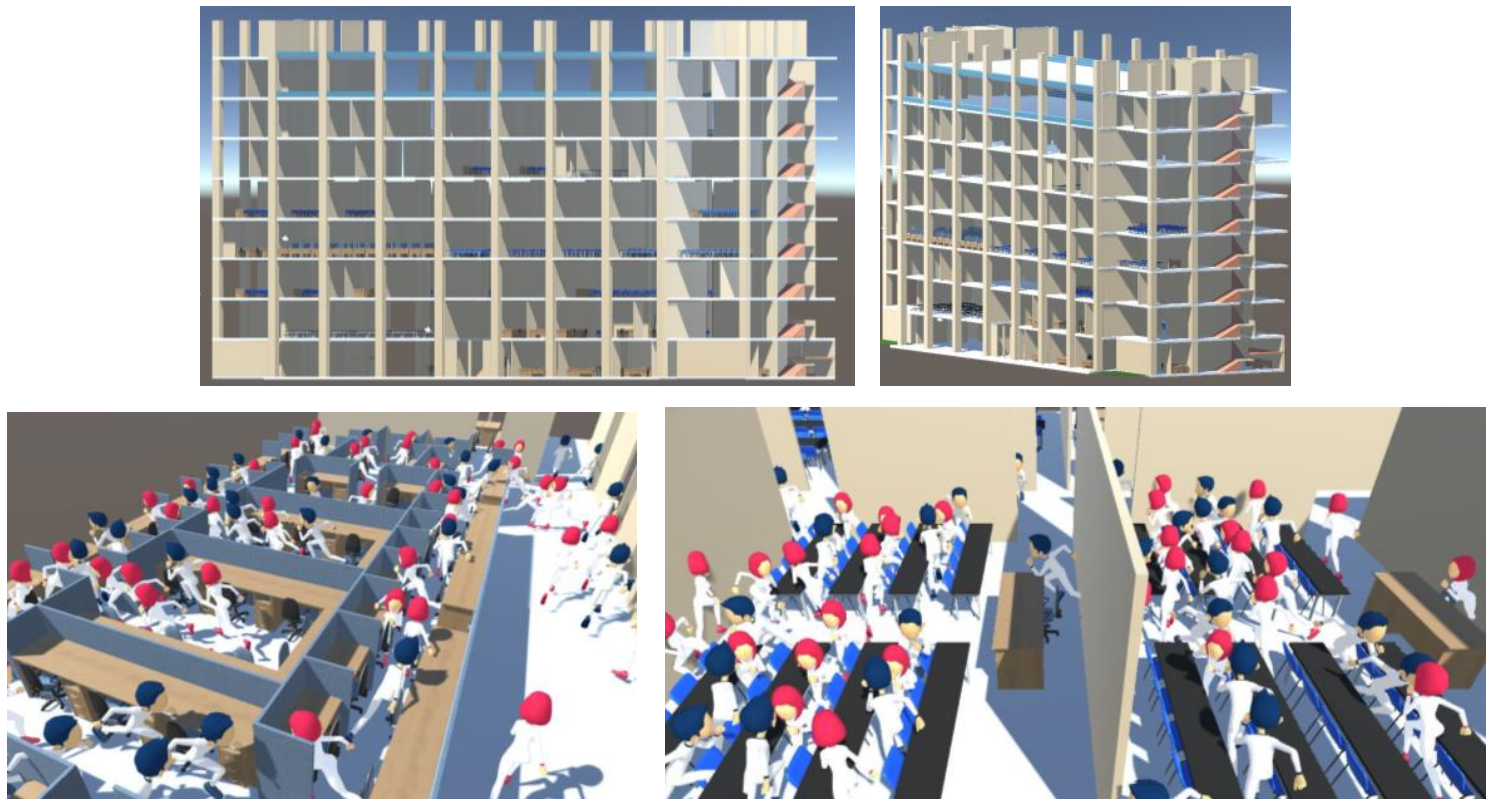

Figure 3. The nine-storey university building, an office area (lower left) and classrooms (lower right) as visualized in Unity

Figure 4 illustrates an evacuation queue from a preliminary simulation run using an initial version of the agents with each one being aware of the exit locations. The next version of the implementation will apply the results of the responses from the earthquake evacuation survey to create a prototype of the agent conceptual framework in Figure 2.

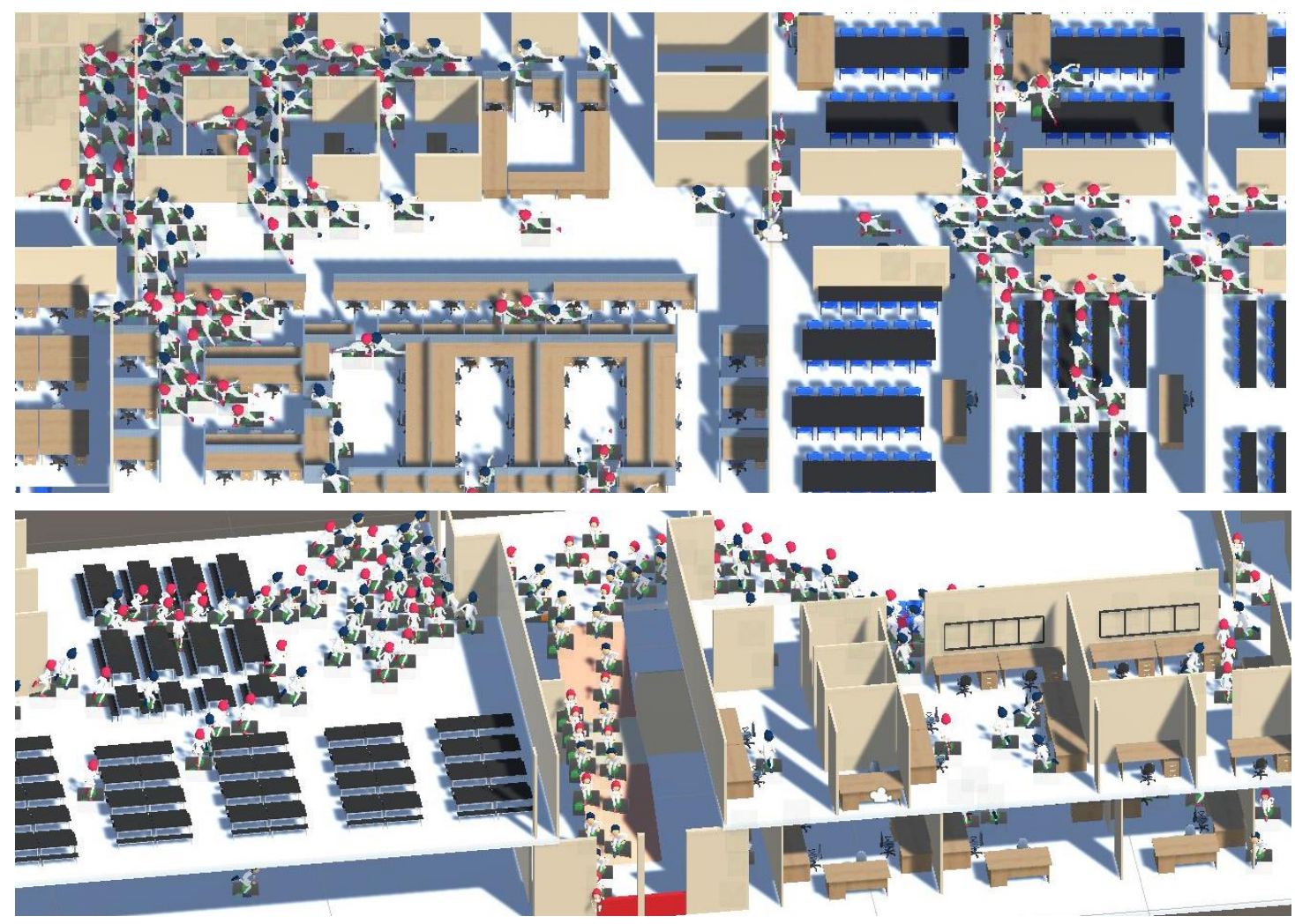

Figure 4. Preliminary Evacuation Simulations in Unity

For each timestamp in seconds, the simulation engine stores information per agent including a generated unique number ID, agent's role (e.g., faculty, staff, student), current location (e.g., floor 1, stairs, parking lot), $3 \mathrm{D}$ coordinates $(\mathrm{x}, \mathrm{y}$, and $\mathrm{z}$ ) and the evacuation state (e.g., false if the agent has not yet arrived at the 
assembly area). Table 2 shows some rows of a data $\log$ for a simulation run. Table 3 summarizes the evacuation simulation and performance outputs such as graphs and maps. The evacuee count vs time and evacuee floor level vs time graphs, and the evacuee route and heat maps will be produced by the evacuation simulation component while the proposed evacuee schedule and route assignments will be produced by the decision support component. The proposal for the evacuee population profile composition will be based on the experiments that will be conducted focusing on how behavioural modification can improve evacuation performance.

Table 2. Evacuation Simulation Data Log

\begin{tabular}{cccccccc}
\hline $\begin{array}{c}\text { Time } \\
\text { (seconds) }\end{array}$ & Agent ID & $\begin{array}{c}\text { Agent } \\
\text { Role }\end{array}$ & Location & x-Coord & $\begin{array}{c}\text { y-Coord } \\
\text { (elevation) }\end{array}$ & z-Coord & $\begin{array}{c}\text { Evacuation } \\
\text { State }\end{array}$ \\
\hline 2.0 & 94172 & Student & Floor 2 & 22.03 & 3.50 & 10.32 & False \\
2.0 & 90032 & Student & Floor 2 & 22.76 & 3.50 & 11.50 & False \\
2.0 & 83148 & Staff & Floor 2 & 33.99 & 3.50 & 8.57 & False \\
\hline
\end{tabular}

Table 3. Evacuation Simulation and Performance Outputs

Evacuee Count vs Time and Evacuee Floor Level vs Time graphs

Evacuee Route and Heat Maps

Proposed Evacuee Schedule and Route Assignments

Proposed Evacuee Population Profile will not only track the progress of the evacuation but will also provide additional indications of congestions (e.g., trendline not continuous and gradually decreasing)

will illustrate bottlenecks and other crucial phenomena to allow further observations and analysis for evacuation planning

will serve as a planning guide for the authorities and decision-makers of the study site to improve evacuation performance

will show the results of the analysis from experiments illustrating scenarios of varying composition of the evacuee archetypes obtained from the cluster analysis

\section{CONCLUSION AND FUTURE WORK}

Human behavioural modelling is a critical component of evacuation simulation. The aspects of behaviour involved in this study, which are expected to affect evacuation performance, include experience, personality, emotions and decision-making. Cluster analysis and SEM are valuable methods in creating the human agent model by integrating findings from literature and survey results and generating a set of statistics to evaluate and produce the most suitable model. SG enhances the effectiveness of evacuation simulation through its inherent user interaction capabilities. As an SG platform, Unity is indeed a powerful and accessible tool for the research community due to its being an open-source software and its effective packages at very reasonable cost.

The succeeding version of the implementation will apply the results of the earthquake evacuation survey on the agent model and perform the simulations on the four presented scenarios.

\section{ACKNOWLEDGEMENTS}

This study is being funded by the Swinburne University of Technology and the CSIRO Data61 Business Unit, both based in Australia. Additionally, the authors acknowledge the National University Manila, Philippines for providing the necessary datasets and assistance.

\section{REFERENCES}

Chen, Y., 2015. A Serious Game -- Defying Disaster -- Earthquake. Worcester Polytechnic Institute.

Choi, S., Do, M., 2019. Effect of Evacuation Information and Evacuation Behaviors on High-Rise Apartment Residents. KSCE J. Civ. Eng. 23, 4886-4897. https://doi.org/10.1007/s12205-019-2126-3

Chu, M.L., Parigi, P., Law, K.H., Latombe, J.-C., 2015. Simulating individual, group, and crowd behaviors in building egress. Simulation 91, 825-845. https://doi.org/10.1177/0037549715605363 
Feng, Z., González, V.A., Amor, R., Spearpoint, M., Thomas, J., Sacks, R., Lovreglio, R., Cabrera-Guerrero, G., 2020. An immersive virtual reality serious game to enhance earthquake behavioral responses and post-earthquake evacuation preparedness in buildings. Adv. Eng. Informatics 45, 101118. https://doi.org/https://doi.org/10.1016/j.aei.2020.101118

García-García, C., Larios-Rosillo, V., Luga, H., 2013. Agent Behaviour Modeling Using Personality Profile Characterization for Emergency Evacuation Serious Games, in: Plemenos, D., Miaoulis, G. (Eds.), Intelligent Computer Graphics 2012. Springer Berlin Heidelberg, Berlin, Heidelberg, pp. 107-128. https://doi.org/10.1007/978-3-642-31745-3_6

Gwynne, S., Amos, M., Kinateder, M., Benichou, N., Boyce, K., van Der Wal, C.N., Ronchi, E., 2020. The future of evacuation drills: Assessing and enhancing evacuee performance. Saf. Sci. 129, 104767.

Gwynne, S.M. V, Kuligowski, E.D., Boyce, K.E., Nilsson, D., Robbins, A.P., Lovreglio, R., Thomas, J.R., Roy-Poirier, A., 2017. Enhancing egress drills: Preparation and assessment of evacuee performance. Fire Mater. 43, 613-631.

Haghani, M., 2020. Optimising crowd evacuations: Mathematical, architectural and behavioural approaches. Saf. Sci. 128, 104745. https://doi.org/https://doi.org/10.1016/j.ssci.2020.104745

Kiyono, J., Miura, F., Takimoto, K., 1996. Simulation of emergency evacuation behaviour during disaster by using distinct element method. Doboku Gakkai Ronbunshu 1996, 233-244.

Littlejohn, S.W., Foss, K.A., 2005. Theories of human communication. Thomson Wadsworth, Canada.

Low, S.M., Lawrence-Zúñiga, D., 2003. The anthropology of space and place: Locating culture. Blackwell Pub.

Mohler, B.J., Thompson, W.B., Creem-Regehr, S.H., Pick, H.L., Warren, W.H., 2007. Visual flow influences gait transition speed and preferred walking speed. Exp. brain Res. 181, 221-228.

Ni, L., Gonzalez, V., Liu, J., Rahouti, A., Zhang, L., Taing, B.P., Miller, T., Oren, N., Sakurai, Y., Noda, I., Savarimuthu, B.T.R., Cao Son, T., 2018. An Agent-Based Approach to Simulate Post-earthquake Indoor Crowd Evacuation. Springer International Publishing, Cham, pp. 568-575.

Rahman, N.A., 2019. Crowd Behavior Simulation of Pedestrians During Evacuation Process: DEM-Based Approach. Springer Singapore.

Rendón Rozo, K., Arellana, J., Santander-Mercado, A., Jubiz-Diaz, M., 2019. Modelling building emergency evacuation plans considering the dynamic behaviour of pedestrians using agent-based simulation. Saf. Sci. 113, 276-284. https://doi.org/https://doi.org/10.1016/j.ssci.2018.11.028

Şahin, C., Alhajj, R., 2020. Crowd Behavior Modeling in Emergency Evacuation Scenarios Using BeliefDesire-Intention Model, in: Kaya, M., Birinci, Ş., Kawash, J., Alhajj, R. (Eds.), Putting Social Media and Networking Data in Practice for Education, Planning, Prediction and Recommendation. Springer International Publishing, Cham, pp. 1-14. https://doi.org/10.1007/978-3-030-33698-1_1

Song, Y., Xie, K., Su, W., 2019. Mechanism and strategies of post-earthquake evacuation based on cellular automata model. Int. J. Disaster Risk Reduct. 34, 220-231. https://doi.org/https://doi.org/10.1016/j.ijdrr.2018.11.020

Sun, Q., Turkan, Y., Mutis, I., Hartmann, T., 2019. A BIM Based Simulation Framework for Fire Evacuation Planning. Springer International Publishing, Cham, pp. 431-438.

Velasco, J.N., Tirao, J.L.M., Enoria, A.S., San Juan, S.Q., Ubaldo, H.F., 2019. Liquefaction potential mapping of the city of Valenzuela, Philippines, in: 2019 IEEE 11th International Conference on Humanoid, Nanotechnology, Information Technology, Communication and Control, Environment, and Management (HNICEM). IEEE, pp. 1-4.

Wąs, J., Lubaś, R., Myśliwiec, W., 2012. Proxemics in Discrete Simulation of Evacuation, in: Sirakoulis, G.C., Bandini, S. (Eds.), Cellular Automata. Springer Berlin Heidelberg, Berlin, Heidelberg, pp. 768775 .

Zoumpoulaki, A., Avradinis, N., Vosinakis, S., Konstantopoulos, S., Perantonis, S., Karkaletsis, V., Spyropoulos, C.D., Vouros, G., 2010. A Multi-agent Simulation Framework for Emergency Evacuations Incorporating Personality and Emotions. Artif. Intell. Theor. Model. Appl. 